Published in final edited form as:

Dig Dis Sci. 2012 May ; 57(5): . doi:10.1007/s10620-012-2035-5.

\title{
Pharmacogenetics of the Effects of Colesevelam on Colonic Transit in Irritable Bowel Syndrome with Diarrhea
}

\author{
Banny S. Wong, \\ Clinical Enteric Neuroscience Translational and Epidemiological Research (CENTER), Mayo \\ Clinic, Charlton, 8-110, 200 First Street S.W., Rochester, MN 55905, USA \\ Michael Camilleri, \\ Clinical Enteric Neuroscience Translational and Epidemiological Research (CENTER), Mayo \\ Clinic, Charlton, 8-110, 200 First Street S.W., Rochester, MN 55905, USA \\ Paula J. Carlson, \\ Clinical Enteric Neuroscience Translational and Epidemiological Research (CENTER), Mayo \\ Clinic, Charlton, 8-110, 200 First Street S.W., Rochester, MN 55905, USA \\ Suwebatu Odunsi-Shiyanbade, \\ Clinical Enteric Neuroscience Translational and Epidemiological Research (CENTER), Mayo \\ Clinic, Charlton, 8-110, 200 First Street S.W., Rochester, MN 55905, USA
}

\section{Sanna McKinzie,}

Clinical Enteric Neuroscience Translational and Epidemiological Research (CENTER), Mayo

Clinic, Charlton, 8-110, 200 First Street S.W., Rochester, MN 55905, USA

\section{Irene Busciglio,}

Clinical Enteric Neuroscience Translational and Epidemiological Research (CENTER), Mayo Clinic, Charlton, 8-110, 200 First Street S.W., Rochester, MN 55905, USA

Duane Burton, and

Clinical Enteric Neuroscience Translational and Epidemiological Research (CENTER), Mayo Clinic, Charlton, 8-110, 200 First Street S.W., Rochester, MN 55905, USA

\section{Alan R. Zinsmeister \\ Division of Biomedical Statistics and Informatics, Department of Health Sciences Research, College of Medicine, Mayo Clinic, Rochester, MN, USA}

Michael Camilleri: camilleri.michael@mayo.edu

\begin{abstract}
Background-Protein products of klotho $\beta(K L B)$ and fibroblast growth factor receptor 4 (FGFR4) impact fibroblast growth factor 19-mediated feedback inhibition of hepatic bile acid (BA) synthesis. Variants of $K L B$ and FGFR4 influence colonic transit (CT) in diarrheapredominant irritable bowel syndrome (IBS-D).
\end{abstract}

Aim-The purpose of this study was to test the hypothesis that colesevelam's slowing effects on CT in IBS-D patients is influenced by genetic variants in $K L B$ and FGFR4.

(C) Springer Science+Business Media, LLC 2012

Correspondence to: Michael Camilleri, camilleri.michael@mayo.edu.

Conflict of interest The authors have no conflicts of interest to disclose. 
Methods-We examined pharmacogenetic effects of $K L B$ and $F G F R 4$ coding variants (SNPs) on scintigraphic $\mathrm{CT}$ response to the BA sequestrant, colesevelam $1.875 \mathrm{~g}$ b.i.d. versus placebo (PLA) for 14 days in 24 female IBS-D patients.

Results-FGFR 4 rs 351855 and $K L B$ rs 497501 were associated with differential colesevelam effects on ascending colon (AC) half-emptying time ( $t_{1 / 2}, P=0.046$ and $P=0.085$ respectively) and on overall CT at $24 \mathrm{~h}$ (geometric center, GC24: $P=0.073$ and $P=0.042$, respectively), with slower transit for rs351855 GA/AA (but not for GG) and rs497501 CA/AA (but not CC) genotypes.

Conclusion-FGFR 4 rs 351855 and $K L B$ rs 4975017 SNPs may identify a subset of IBS-D patients with beneficial response to colesevelam.

\section{Keywords}

Bile acid; Klotho $\beta$, FGFR4

\section{Background}

A crucial mechanism in the control of bile acid (BA) homeostasis involves the fibroblast growth factor 19 (FGF19)-mediated feedback inhibition of CYP7A1, the rate-limiting enzyme in the hepatic synthesis of BA from cholesterol. This negative feedback requires binding of the hormone FGF19 to the receptor fibroblast growth factor receptor 4 (FGFR4), and its co-receptor klotho $\beta(\mathrm{KLB})$ on the hepatocytes plasma membrane [1]. Walters et al. [2] showed that patients with BA malabsorption (BAM) have low serum FGF-19 levels, suggesting that impaired FGF19-mediated negative feedback leads to excessive hepatic BA synthesis and secretion into the small intestine. Without increased ileal BA absorption through the apical sodium-dependent BA transporter (ASBT, also called ileal BA transporter or IBAT), this excess BA leaks into the colon, inducing colonic secretion and motility which manifest as diarrhea.

Bile acid malabsorption is observed in around $30 \%$ of chronic functional diarrhea that is otherwise unexplained [3]. This is based on measurements such as ${ }^{75}$ SeHCAT retention [4] as well as positive response to trials of BA binding therapy. When measurements of fecal BAs or ${ }^{75} \mathrm{SeHCAT}$ retention are not available, an empiric trial with a BA binder such as cholestyramine is commonly used to "diagnose" BAM based on therapeutic response to therapy. In patients with clinically diagnosed diarrhea-predominant irritable bowel syndrome (IBS-D), Odunsi-Shiyanbade et al. [5] showed that the BA sequestrant, colesevelam, reduces colonic transit. The reduction in colonic transit by colesevelam is greatest in patients with high serum $7 a \mathrm{C} 4$, a surrogate marker of hepatic $\mathrm{BA}$ synthesis rate previously validated against the ${ }^{75} \mathrm{SeHCAT}$ retention test [6].

We have previously demonstrated that genetic variants of $K L B$ and FGFR4, whose protein products are critical to FGF19-mediated feedback inhibition of hepatic BA synthesis, significantly influence baseline colonic transit in IBS-D [7]. We also observed that genetic variants in $K L B$ and FGFR4 influence the acceleration of colonic transit by treatment with exogenous BA in IBS-C patients [8]. In this study, we aimed to test the hypothesis that colesevelam's slowing effects on colonic transit in IBS-D patients is influenced by genetic variants in $K L B$ and $F G F R 4$. In the present study, participants were not selected on the basis of evidence of BAM. 


\section{Methods}

\section{Study Design}

We performed a pharmacogenetic analysis of data acquired in a previously published double-blind, randomized study [5] of oral placebo versus colesevelam, $1.875 \mathrm{~g}$, twice daily, taken for 12-14 consecutive days, in 24 female IBS-D patients (mean age 42.7 years). Patients met Rome II criteria for IBS.

\section{Participants}

The detailed information about these participants, recruitment and trial flow are described in the previous publication [5] and summarized in Fig. 1.

\section{Colonic Transit}

Colonic transit was measured by a validated scintigraphic method using a delayed-release capsule to deliver ${ }^{111} \mathrm{In}$-charcoal particles to the ileocolonic region $[9,10]$. The primary endpoints for $\mathrm{CT}$ were ascending colon emptying half-time (AC $t_{1 / 2}$ ) and geometric center at 24 h (GC24), a validated measure of overall colonic transit.

\section{Genotyping}

Genotyping was performed on five common, non-synonymous, single nucleotide polymorphisms (SNPs) in KLB (rs17618244 and rs497501) and FGFR4 (rs351855, rs386618, and rs1966265), as shown in Table 1 [7]. Minor allele frequencies were $>15 \%$ for all five SNPs. Genomic DNA was isolated from peripheral blood samples (usually drawn from a forearm vein) using standard methods; the blood draw was obtained at the screening visit, prior to any drug administration. TaqMan SNP assay was used for genotyping as previously described [7]. A single subject in the colesevelam treatment group did not give a blood sample, so genotype data were not available for this subject.

\section{Statistical Analysis}

An analysis of covariance was used to test for differential treatment effects on CT by colesevelam across genotype groups using a dominant genetic model coding for genotype (homozygotes of major allele vs. heterozygotes plus homozygotes of minor allele). The ANCOVA models included terms for genotype (the covariate), treatment (colesevelam vs. placebo) and the "interaction term" of genotype by treatment. This latter term is used to test for differences in treatment effects depending on genotype. All reported $P$ values are unadjusted for multiple genes assessed, or multiple specific pair-wise (treatment group) comparisons.

\section{Results}

Hardy-Weinberg equilibrium (HWE) was assessed in healthy control patients in a previous study (Table 1; [7]). HWE was satisfied in four of the five candidate SNPs in this study, with the exception of FGFR 4 rs376618. However, there were no significant differential treatment effects associated with this SNP. The distribution of participants' genotypes for the four other SNPs is shown in Table 2.

\section{Effect of FGFR4 Genotype Variation}

The functional genetic variant, FGFR 4 rs 351855 (Gly388Arg), was associated with differential colesevelam treatment effect on AC $t_{1 / 2}(P=0.046)$ and for GC24 $(P=0.073)$. In the rs351855 GA/AA genotype group, colesevelam significantly delayed CT, with increased AC $t_{1 / 2}(23.46 \pm 3.56 \mathrm{~h}$ vs. $9.95 \pm 2.70 \mathrm{~h}$ on placebo, $P=0.04)$ and decreased 
GC24 (2.28 $=0.31$ vs. $3.59=0.56$ units on placebo, $P=0.05)$. In contrast, in the rs351855

GG genotype group, there was no significant effect of colesevelam treatment on CT with either AC $t_{1 / 2}(13.38 \pm 2.79 \mathrm{~h}$ vs. $18.50 \pm 5.33 \mathrm{~h}$ on placebo, $P=0.43)$ or GC24 (3.49 \pm 0.59 vs. $3.10 \pm 0.40$ units on placebo, $P=0.56$, see Fig. 2 ). No significant differential colesevelam treatment effects were detected in the two other FGFR4 SNPs tested.

\section{Effect of $K L B$ Genotype Variation}

There was a differential treatment effect on CT associated with $K L B$ rs4975017 $(P=0.048)$. In the CA/AA genotype group, colesevelam treatment compared to placebo was associated with lower GC24 value $(P=0.042)$, and a numerically longer in $\mathrm{AC} t_{1 / 2}(P=0.085)$ on colesevelam versus placebo (Fig. 3). No such significant treatment effects were observed in the group with CC genotype ( $P>0.30$ for both GC 24 and $\left.\mathrm{AC} t_{1 / 2}\right)$.

The functional variant $K L B$ rs17618244 (Arg728Gln) was associated with numerical though not statistically significant differential colesevelam treatment effects on AC $t_{1 / 2}$ and GC24. Modest treatment effects were observed in the GG genotype ( $P=0.14$ for AC $t_{1 / 2}, P=0.12$ for GC24), but not in the GA/AA genotype ( $P>0.8$, see Fig. 4$)$.

\section{Discussion}

We have shown that the polymorphisms FGFR 4 rs351855 and $K L B$ rs4975017 may influence the colonic transit response to colesevelam in female patients with IBS-D. These data provide further evidence that genetic variations in the FGF19-FGFR4-KLB pathway that are critical for feedback inhibition of hepatic BA synthesis may affect colesevelammediated delay of colonic transit in IBS-D. These data also indirectly support the relevance of the FGF19-FGFR4-KLB pathway in the control of colonic transit.

We have investigated the association of genotype with potential differential treatment effects on colonic transit in patients with IBS, i.e. the impact of pharmacogenetics on the transit responses to treatment of IBS-C patients.

First, the GG genotype of $K L B$ SNP rs 17618244 is associated with accelerated colonic transit [7]. FGFR4 SNPs appear to be functioning, since the FGFR4 SNPs modulate the actions of KLB. Thus, we showed KLB rs17618244, with either FGFR 4 rs 351855 or FGFR4 rs1966265, influenced colonic transit in IBS-D patients in the absence of any pharmacological therapy [7].

Second, in a separate pharmacogenomics study, we also showed that genetic variants in $K L B$ and FGFR 4 influence the degree of acceleration of colonic transit in IBS-C patients mediated by treatment with chenodeoxycholate (CDC), a primary bile salt [8]. For example, in IBS-C patients, $K L B$ rs $17618244 \mathrm{GG}$ genotype was associated with accelerated AC $t_{1 / 2}$ with 500 and 1,000 mg CDC doses, whereas GA/AA genotype showed accelerated transit with $1,000 \mathrm{mg}$, but not with the $500 \mathrm{mg}$ CDC [8].

The present study has shown that, in specific genetic subgroups, there is a very significant effect on colonic transit of colesevelam compared to placebo. This effect is manifest in the overall assessment of colonic transit with the geometric center measurement at $24 \mathrm{~h}$, as well as with prolongation in the emptying time of the ascending colon, which would allow greater time for absorption of water in the proximal colon and change in stool frequency and consistency. We have previously demonstrated [10] that a 1 geometric center unit difference at $24 \mathrm{~h}$ corresponds to a $\sim 0.58$ unit change in stool consistency as measured by the Bristol stool form scale and a change in stool frequency of 0.44/day. In addition, the magnitude of difference in $\mathrm{AC} t_{1 / 2}$ observed with colesevelam compared to placebo (difference in mean 
values $\sim 13 \mathrm{~h}$ ) is greater than the effect of alosetron (difference in mean values $5.2 \mathrm{~h}$ ) in AC $t_{1 / 2}$ in patients with IBS-D [11].

Our analysis of the genotype-intermediate phenotype association did not correct for the five tested gene variations and, therefore, the data are hypothesis-generating and require replication. Nevertheless, the results are quite remarkable, as they are based on a wellcharacterized intermediate phenotype (colonic transit measurement) and on a sample of only 12 patients treated with colesevelam and 12 treated with placebo. These data are also helpful, since they provide the basis for calculating the sample size required in future replication studies.

The three other SNPs were non-contributory in this association analysis of the effect of colesevelam on colonic transit. This may reflect the fact that FGFR4 rs351855 and $K L B$ rs4975017 had the highest MAFs of 30-40\% among the five SNPs studied, whereas the three other SNPs had MAF of only 18-23\%. Thus, the lack of association with other SNPs may reflect a type II error. This is particularly relevant to the assessment of the association of $K L B$ rs17618244 with the effects of colesevelam on colonic transit and AC emptying, as shown in Fig. 4. For $K L B$ rs 17618244, the distribution of genotype was GG for each GA/ $\mathrm{AA}$, and the randomization yielded roughly balanced subgroups in each treatment. The difference (colesevelam vs. placebo) in GC24 values was essentially zero in the GA/AA genotype, while it was $\sim 0.9$ in the GG genotype. Thus, if the treatment effect in a future study were to replicate the mean data observed in the current study, that is, a differential treatment effect on colonic transit (GC24) of 0.1 in GA/AA group (e.g. 3.2 vs. 3.1), and 1.3 in the GG group (e.g. 3.3 vs. 2.0), 176 subjects would be needed to document a pharmacogenetic effect of this SNP on response to colesevelam with $80 \%$ power. Thus, while we cannot exclude a type II error in assessing the effects of the three non-significant SNPs, their impact seems unlikely to be clinically relevant.

Bile acid malabsorption is increasingly recognized as a cause of chronic, functional diarrhea which is otherwise unexplained. It is also considered that BAM contributes to diarrhea in patients with IBS-D, since there is significant overlap of symptoms of functional diarrhea and IBS-D, and BA binding with colesevelam retardation of colonic transit was significantly associated with BA synthesis, measured by the surrogate serum $7 a \mathrm{C} 4$ [4]. Given the claim that $\sim 30 \%$ of patients with chronic functional diarrhea [3] and $\sim 20 \%$ of patients with IBS$\mathrm{D}$ [12] have evidence of BAM, it is clinically relevant to screen for BAM in patients with symptoms of functional diarrhea or IBS-D [2, 3, 13]. For example, these prevalence rates are much higher than the estimated $0.4-4 \%$ of such patients who have celiac disease [14-16]. Given the observations in our current study, we believe that, in addition to screening for BAM in patients presenting with IBS-D or functional diarrhea, it is appropriate to include a therapeutic trial of a BA-binding medication. Larger studies are needed to determine whether the genotype assay would help identify optimal responders to this form of therapy.

The current screening tests for BAM are measurement of fecal BAs and the ${ }^{75} \mathrm{SeHCAT}$ retention test. Regrettably, fecal BA measurements are not widely available, and the ${ }^{75} \mathrm{SeHCAT}$ retention test is not available in the United States as the isotope was never approved for clinical testing. Our data suggest that the FGFR4 rs351855 and KLB rs4975017 polymorphisms may facilitate identification of a subset of IBS-D patients with significant beneficial response to BA sequestration with colesevelam.

\section{Acknowledgments}

We thank Mary Lempke, Pharm. D., research pharmacist, and Cindy Stanislav, secretary, for assistance. This work is funded by grant RO1-DK092179 from the National Institutes of Health to Dr. Camilleri and by Mayo Clinic CTSA grant (RR24150). 


\section{References}

1. Hofmann AF, Mangelsdorf DJ, Kliewer SA. Chronic diarrhea due to excessive bile acid synthesis and not defective ileal transport: a new syndrome of defective fibroblast growth factor 19 release. Clin Gastroenterol Hepatol. 2009; 7:1151-1154. [PubMed: 19665580]

2. Walters JR, Tasleem AM, Omer OS, Brydon WG, Dew T, le Roux CW. A new mechanism for bile acid diarrhea: defective feedback inhibition of bile acid biosynthesis. Clin Gastroenterol Hepatol. 2009; 7:1189-1194. [PubMed: 19426836]

3. Wedlake L, A'Hern R, Russell D, Thomas K, Walters JR, Andreyev HJ. Systematic review: the prevalence of idiopathic bile acid malabsorption as diagnosed by SeHCAT scanning in patients with diarrhoea-predominant irritable bowel syndrome. Aliment Pharmacol Ther. 2009; 30:707-717. [PubMed: 19570102]

4. Nyhlin H, Merrick MV, Eastwood MA, Brydon WG. Evaluation of ileal function using 23selena-25-homotaurocholate, a-gamma-labeled conjugated bile acid. Initial clinical assessment. Gastro-enterology. 1983; 84:63-68.

5. Odunsi-Shiyanbade ST, Camilleri M, McKinzie S, et al. Effects of chenodeoxycholate and a bile acid sequestrant, colesevelam, on intestinal transit and bowel function. Clin Gastroenterol Hepatol. 2010; 8:159-165. [PubMed: 19879973]

6. Brydon WG, Nyhlin H, Eastwood MA, Merrick MV. Serum 7 alpha-hydroxy-4-cholesten-3-one and selenohomocholyltaurine (SeHCAT) whole body retention in the assessment of bile acid induced diarrhoea. Eur J Gastroenterol Hepatol. 1996; 8:117-123. [PubMed: 8723414]

7. Wong BS, Camilleri M, Carlson PJ, et al. A klotho $\beta$ variant mediates protein stability and associates with colon transit in irritable bowel syndrome with diarrhea. Gastroenterology. 2011; 140:19341942. [PubMed: 21396369]

8. Rao AS, Wong BS, Camilleri M, et al. Chenodeoxycholate in females with irritable bowel syndrome-constipation: a pharma-codynamic and pharmacogenetic analysis. Gastroenterology. 2010; 139:1549-1558. [PubMed: 20691689]

9. Camilleri M. Scintigraphic biomarkers for colonic dysmotility. Clin Pharmacol Ther. 2010; 87:748753. [PubMed: 20410880]

10. Deiteren A, Camilleri M, Bharucha AE, et al. Performance characteristics of scintigraphic colon transit measurement in health and irritable bowel syndrome and relationship to bowel functions. Neurogastroenterol Motil. 2010; 22:415-423. [PubMed: 20025675]

11. Viramontes BE, Camilleri M, McKinzie S, Pardi DS, Burton D, Thomforde GM. Gender related differences in slowing colonic transit by a 5-HT3 antagonist in subjects with diarrhea-predominant irritable bowel syndrome. Am J Gastroenterol. 2001; 92:2671-2676. [PubMed: 11569693]

12. Camilleri M, Nadeau A, Tremaine WJ, et al. Measurement of serum 7alpha-hydroxy-4cholesten-3-one (or 7alphaC4), a surrogate test for bile acid malabsorption in health, ileal disease and irritable bowel syndrome using liquid chromatography-tandem mass spectrometry. Neurogastroenterol Motil. 2009; 21:734-e43. [PubMed: 19368662]

13. Spiller R, Camilleri M, Longstreth GF. Perspective: do the symptom-based, Rome criteria of irritable bowel syndrome lead to better diagnosis and treatment outcomes? The con argument. Clin Gastroenterol Hepatol. 2010; 8:125-136. [PubMed: 20152787]

14. Sanders DS, Carter MJ, Hurlstone DP, et al. Association of adult coeliac disease with irritable bowel syndrome: a case-control study in patients fulfilling ROME II criteria referred to secondary care. Lancet. 2001; 358:1504-1508. [PubMed: 11705563]

15. El-Salhy M, Lomholt-Beck B, Gundersen D. The prevalence of celiac disease in patients with irritable bowel syndrome. Mol Med Rep. 2011; 4:403-405. [PubMed: 21468583]

16. Korkut E, Bektas M, Oztas E, Kurt M, Cetinkaya H, Ozden A. The prevalence of celiac disease in patients fulfilling Rome III criteria for irritable bowel syndrome. Eur J Intern Med. 2010; 21:389392. [PubMed: 20816591]

Dig Dis Sci. Author manuscript; available in PMC 2013 October 28. 


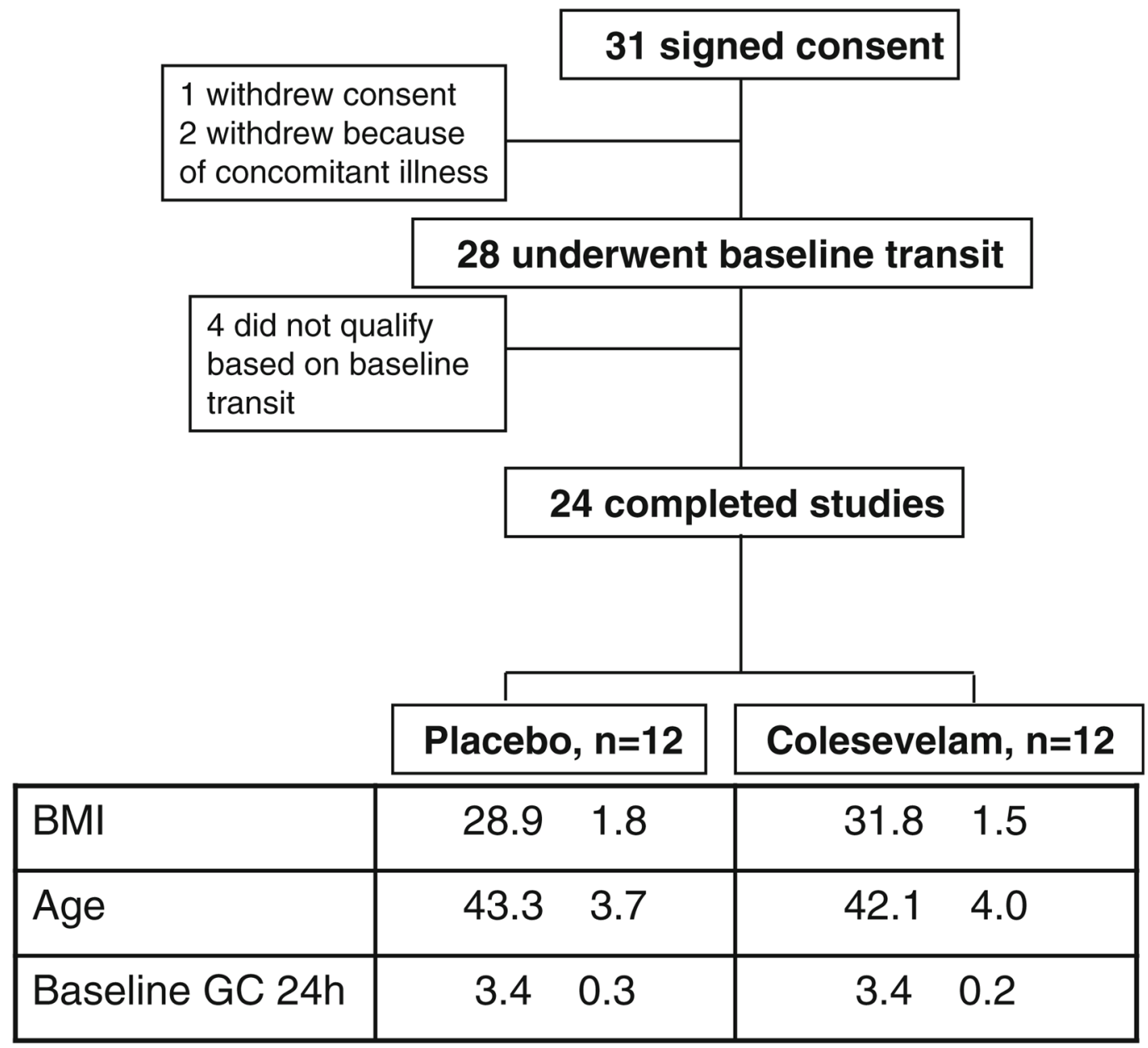

Fig. 1.

Trial flow, participant demographics and baseline features 


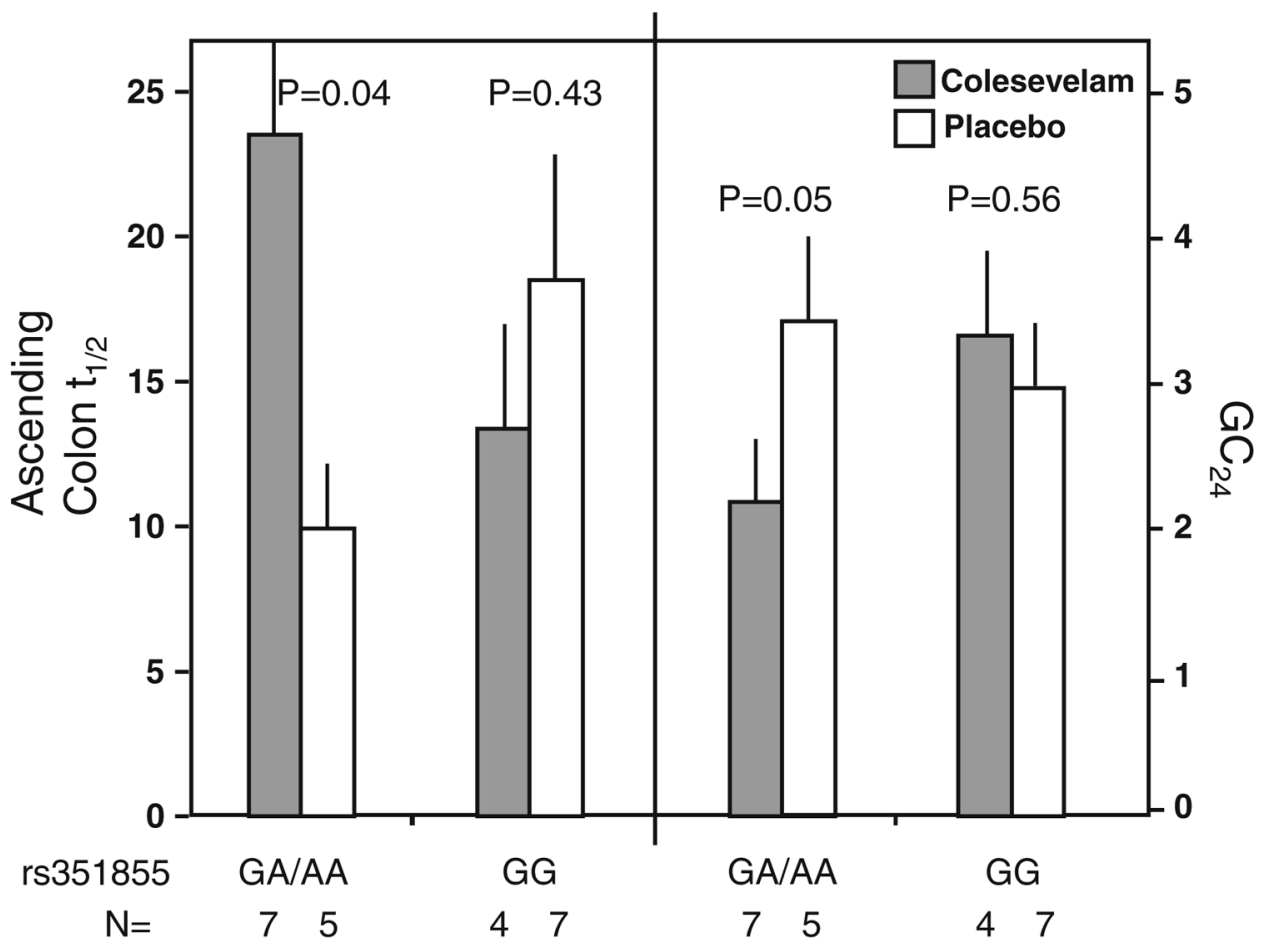

Fig. 2.

Association of FGFR4 variation (SNP rs 351855) and colonic transit response to colesevelam and placebo. The number of participants with specified genotype in each treatment group is provided 


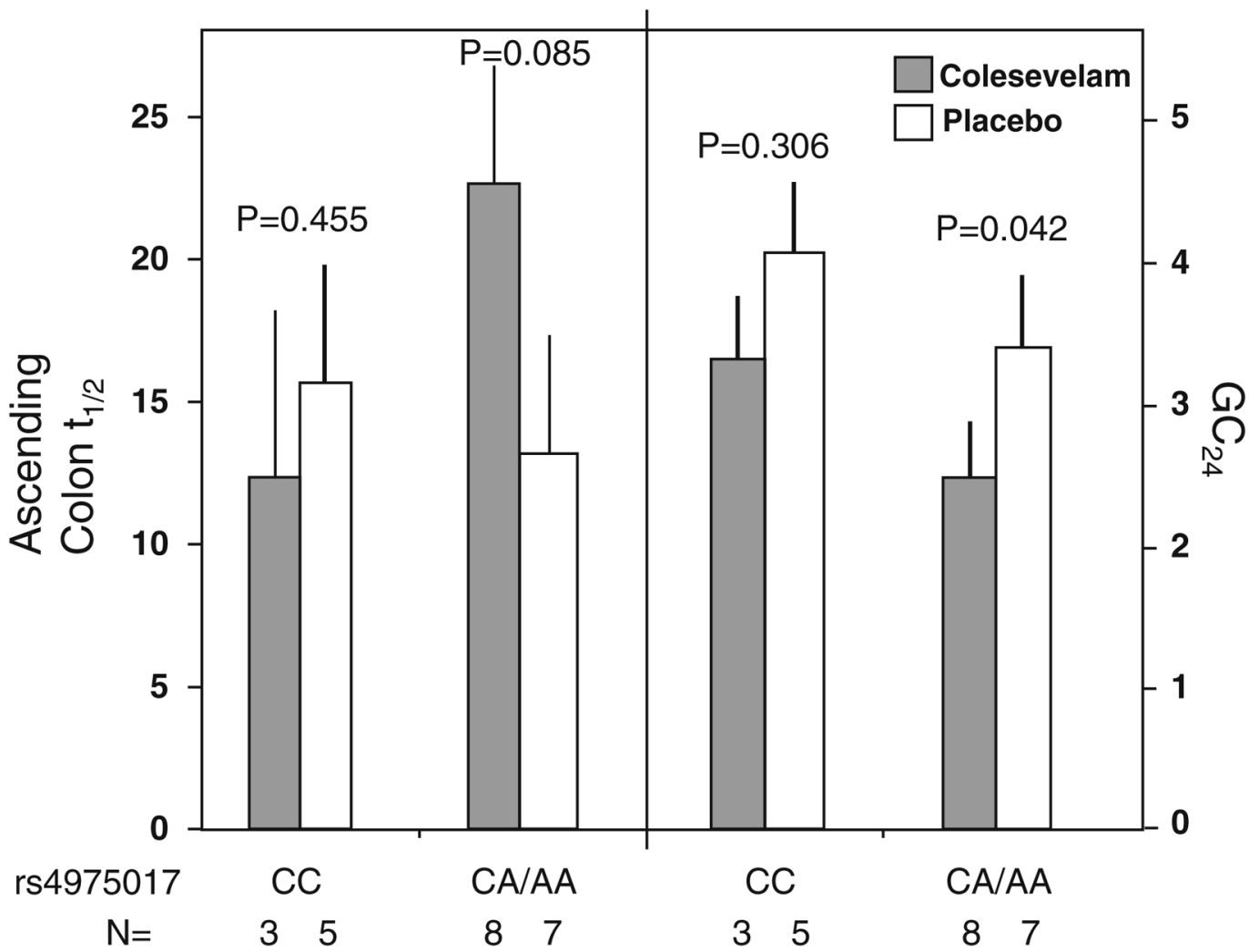

Fig. 3.

Association of KLB variation (SNP rs 4975017) and colonic transit response to colesevelam and placebo. The number of participants with specified genotype in each treatment group is provided 


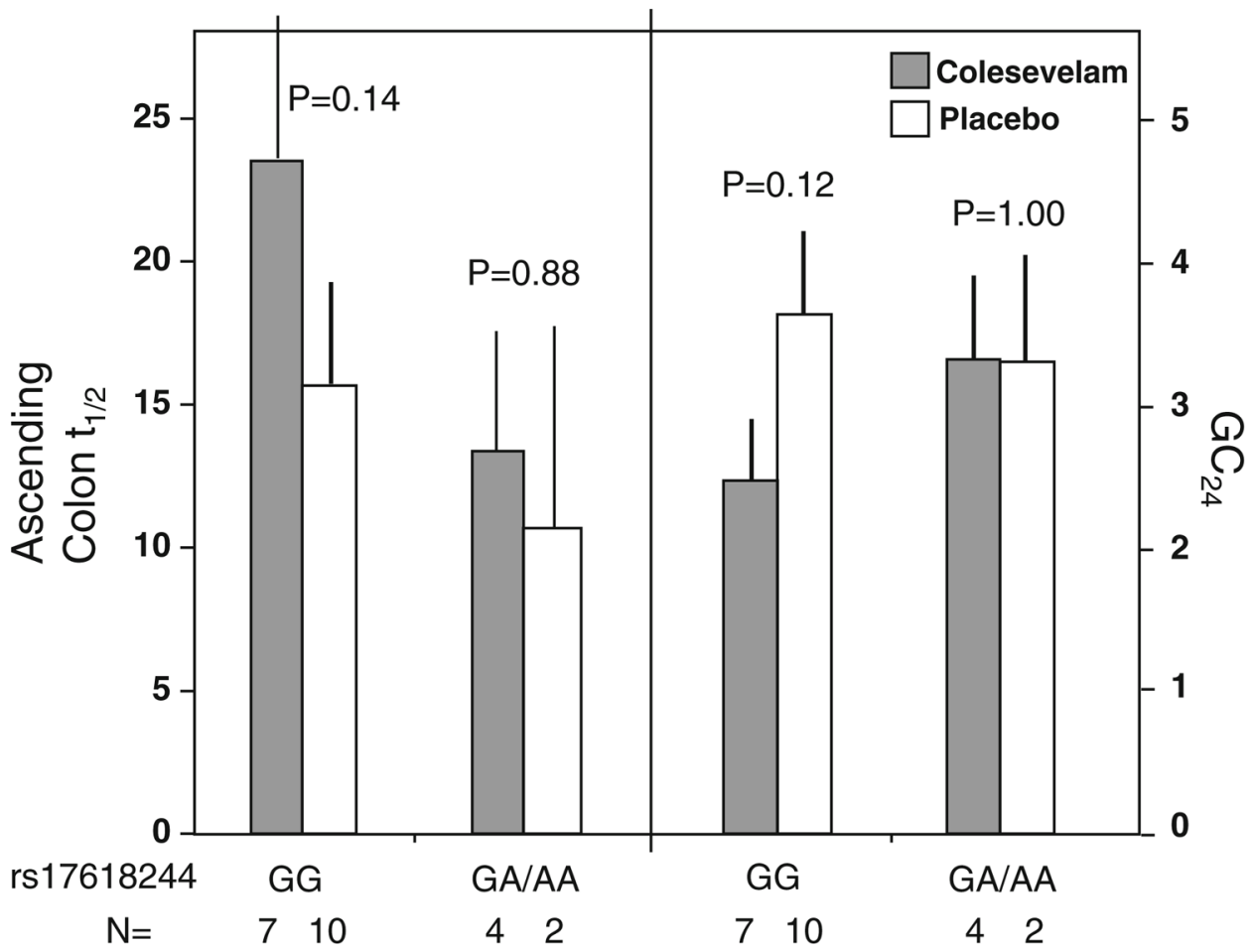

Fig. 4.

Association of KLB rs 17618244 variation and colonic transit response to colesevelam and placebo. The number of participants with specified genotype in each treatment group is provided 


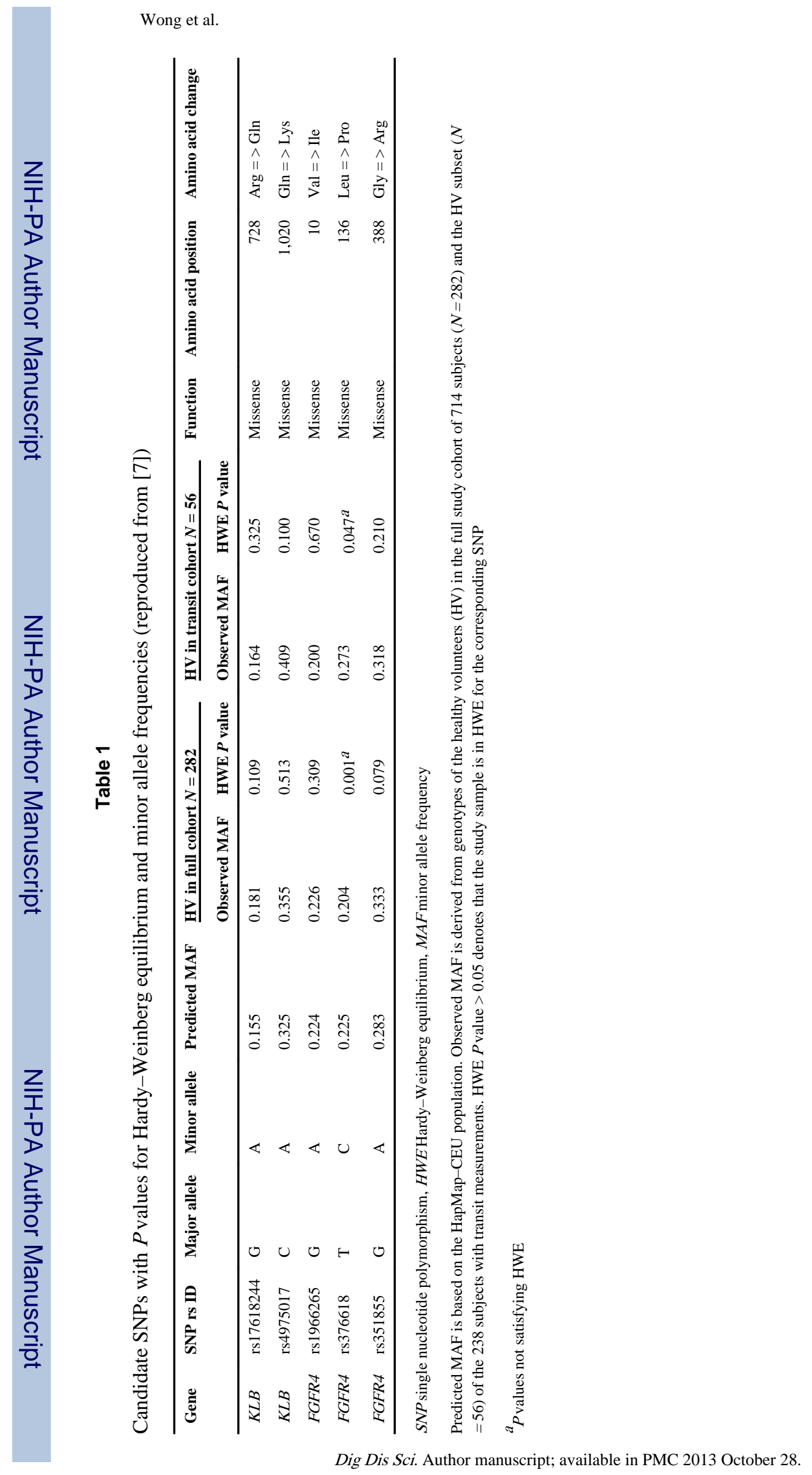

Page 11 
Table 2

Distribution of genotypes in the different treatment groups, separated according to the dominant genetic model

\begin{tabular}{lll}
\hline Genotypes & Placebo & Colesevelam \\
\hline KLB rs17618244 GA/AA: GG & $2: 10$ & $4: 7$ \\
KLB rs4975017 CA/AA: CC & $7: 5$ & $8: 3$ \\
FGFR4 rs1966265 GA/AA: GG & $5: 7$ & $3: 8$ \\
FGFR4 rs351855 GA/AA: GG & $5: 7$ & $7: 4$ \\
\hline
\end{tabular}

